\title{
Associação entre Síndrome de Sjogren e complicações oftalmológicas a partir da avaliação de estudos conforme protocolo STROBE
}

\author{
Association between Sjogren's Syndrome and ophthalmological complications from the \\ evaluation of studies according to the STROBE protocol
}
Asociación entre Síndrome de Sjogren y complicaciones oftalmológicas a partir de la evaluación de estudios según el protocolo STROBE

Renan Monteiro da Silveira ${ }^{1 *}$, Felipe Monteiro Carvalheiro ${ }^{1}$, Thales José Moura Moraes ${ }^{1}$, Ana Clara Monteiro de Araújo ${ }^{1}$, Brenda Nazaré Gomes Andriolo¹.

\section{RESUMO}

Objetivo: Conhecer as taxas de associação entre Sindrome de Sjogren (SS) e suas complicações oculares. Métodos: Estudo transversal analítico. Foram incluídos estudos observacionais, em qualquer idioma, que avaliaram a associação entre SS e complicações oftalmológicas, submetidos desde a criação da base de dados até julho de 2020. Os artigos do PubMed que associaram estas temáticas foram classificados com base nos critérios do protocolo Strengthening the Reporting of Observational Studies in Epidemiology (STROBE), um conjunto de recomendações para melhorar a qualidade dos relatórios de estudos observacionais, em adequado, inadequado e insuficiente. Resultados: A secura ocular foi o sintoma do olho seco com maior associação com SS (98.4\%). Outros mais raramente associadas foram uveítes, inflamação orbital, esclerite, vasculite retiniana e neuromielite óptica. Quanto aos critérios STROBE, os tópicos se enquadraram majoritariamente na categoria "Adequado". Em relação a inadequação, o tópico "Resultados" apresentou maior percentual (16,67\%), assim como na classificação "Insuficiente", juntamente com "Discussão" e "Outras informações" Considerações finais: Há grande heterogeneidade entre os trabalhos analisados. São necessários mais estudos sobre o tema, realizados de maneira padronizada entre as diferentes amostras, para que possa ser feita uma comparação mais fidedigna entre estas.

Palavras-chave: Síndrome de Sjogren, Oftalmologia, Prevalência.

\section{ABSTRACT}

Objective: To know the association rates between Sjogren's Syndrome (SS) and its ocular complications. Methods: Analytical cross-sectional study. Observational studies, in any language, that evaluated the association between SS and ophthalmological complications, included since the creation of the database until July 2020, were included. PubMed articles that associated these themes were classified based on the Strengthening the protocol criteria Reporting of Observational Studies in Epidemiology (STROBE), a set of recommendations to improve the quality of observational study reports, in appropriate, inadequate and insufficient. Results: Dry eye was the symptom of dry eye disease with the highest association with SS (98.4\%). Others more rarely associated were uveitis, orbital inflammation, scleritis, retinal vasculitis and optic neuromyelitis. As for the STROBE criteria, the topics mostly fell into the "Adequate" category. Regarding inadequacy, the topic "Results" presented a higher percentage (16.67\%), as well as in the "Insufficient" classification, together with "Discussion" and "Other information". Final considerations: There is great heterogeneity among the analyzed works. Further studies on the topic are necessary, carried out in a standardized manner among the different samples, so that a more reliable comparison between them can be made.

Keywords: Sjogren's syndrome, Ophthalmology, Prevalence.

1Universidade do Estado do Pará (UEPA), Belém - PA. *E-mail: renanmonteirosilv@gmail.com 


\section{RESUMEN}

Objetivo: Conocer las tasas de asociación entre el Síndrome de Sjogren (SS) y sus complicaciones oculares. Métodos: Estudio analítico transversal. Se incluyeron los estudios observacionales, en cualquier idioma, que evaluaran la asociación entre SS y complicaciones oftalmológicas, incluidos desde la creación de la base de datos hasta julio de 2020. Los artículos de PubMed que asociaban estos temas se clasificaron en base a los criterios de Fortalecimiento del protocolo Reporting of Observational Studies in Epidemiology (STROBE), un conjunto de recomendaciones para mejorar la calidad de los informes de estudios observacionales, en forma apropiada, inadecuada e insuficiente. Resultados: La sequedad ocular fue el síntoma de ojo seco con mayor asociación con SS $(98,4 \%)$. Otros asociados con menor frecuencia fueron uveítis, inflamación orbitaria, escleritis, vasculitis retiniana y neuromielitis óptica. En cuanto a los criterios STROBE, la mayoría de los temas fue encuadrada en la categoría "Adecuado". Con relación a la inadecuación, el tema "Resultados" tuvo un mayor porcentaje (16,67\%), así como en la clasificación "Insuficiente", junto con "Discusión" y "Otra información". Consideraciones finales: Existe una gran heterogeneidad entre los estudios analizados. Son necesarios más investigaciones sobre el tema, realizadas de manera estandarizada entre las diferentes muestras, para que se pueda realizar una comparación más confiable entre ellas.

Palabras clave: Síndrome de Sjogren, Oftalmología, Prevalencia.

\section{INTRODUÇÃO}

A Síndrome de Sjogren (SS) é uma das doenças autoimunes de ocorrência mais comum, sendo frequentemente subdiagnosticada no dia a dia da prática clínica (MAVRAGANI CP, et al., 2019). Apresenta uma prevalência de 0,5 a $1 \%$ da população mundial, afetando predominantemente o sexo feminino na faixa etária da meia idade, com proporção de 9 mulheres para 1 homem. Pode, contudo, ocorrer em qualquer sexo e em qualquer faixa etária (MAVRAGANI CP, et al., 2019). Caracteriza-se por um infiltrado linfocitário que afeta 0 epitélio das glândulas exócrinas, principalmente das glândulas salivares e das glândulas lacrimais (VALIM V, et al., 2015). O principal mecanismo fisiopatológico da doença é a epitelite autoimune, mediada por citocinas e por fatores de crescimento, com consequente destruição e fibrose do parênquima e estruturas das glândulas, provocando prejuízo às suas funções e, consequentemente, acarretando a dimunuição da formação de lágrimas e saliva. (MILANI LMJ, e al., 2017).

Ademais, há hiper-reatividade dos linfócitos $\mathrm{B}$, que se convertem em plasmócitos e produzem anticorpos contra os antígenos do epitélio dos ácinos e dos ductos das glândulas exócrinas. Os Linfócitos T supressores também são atingidos, tendo sua ação diminuída e perpetuando a atividade dos linfócitos $B$ ativados e a agressão tecidual (BETIM FCM, et al., 2016). A Síndrome de Sjogren doença pode se manifestar sozinha, sendo classificada como primária, ou associada a uma doença do tecido conjuntivo de base, sendo classificada como Sindrome de Sjogren secundária. A xeroftalmia e a xerostomia são as manifestações mais prevalentes no quadro clínico. A despeito disso, pode ainda apresentar sintomas sistêmicos de natureza cutânea, hepática, renal, neurológica e vascular, sendo uma doença de múltiplas facetas e de diagnóstico difícil (ALMEIDA JUNIOR ADD, 2019).

A xeroftalmia é a principal manifestação ocular da síndrome e ocorre por meio da diminuição contínua e progressiva do componente aquoso do filme lacrimal, em função da destruição das glândulas lacrimais principais e acessórias pela atividade autoimune. Sintomas oculares, a exemplo de sensação de corpo estranho, queimação, prurido e vermelhidão são comuns e ocorrem já nas fases iniciais da doença. Em consequência da falta de lubrificação, as córneas dos pacientes com SS estão predispostas ao desenvolvimento de defeitos epiteliais crônicos, doenças vasculares e úlceras estéreis ou infectadas. Perfuração ocular pode ocorrer nos casos mais graves, podendo haver perda da integridade ocular e cegueira irreversível (MALLMANN CS, 2015).

Atualmente, os testes e exames mais utilizados no diagnóstico do olho seco e na avaliação da superfície ocular destes pacientes são: biomicroscopia, testes de Schirmer (I, Il e basal), tingimento da superfície ocular com corante Rosa Bengala, fluoresceína ou lisamina verde, avaliação do tempo de ruptura do filme lacrimal, análise bioquímica do filme lacrimal e citologia de impressão da córnea e conjuntiva (ALMEIDA JUNIOR ADD, 2019). 
Para avaliar o impacto da síndrome na qualidade de vida dos portadores e permitir a padronização das queixas dos pacientes, diversos questionários foram elaborados e testados, dentre eles o questionário "Ocular Surface Disease Index" (OSDI) e o questionário "Visual Function Questionnaire" (VFQ-25) (RIBEIRO MVMR, et al., 2019).

A despeito da grande variedade de testes diagnósticos, muitas vezes a doença só é percebida com o atraso de anos, quando o quadro clínico está francamente presente, o que, no contexto da sintomatologia ocular, implica em importante limitação das atividades diárias e em piora da qualidade de vida do paciente (CENDÓN CHAPELA S, 2016).

Embora seja uma enfermidade comum, a subnotificação sofrida pela doença na prática médica deve-se à heterogeneidade e à natureza muitas vezes inespecífica das manifestações clínicas precoces. Acredita-se amplamente que a Síndrome de Sjogren permanece sem diagnóstico em aproximadamente metade casos (AKPEK EK, et al., 2015). Na maioria dos casos, o diagnóstico de SS é atrasado em um período de 6 anos até o de uma década (AKPEK EK, et al., 2019). Esse atraso no diagnóstico é de grande impacto no curso clínico da doença, dado que aproximadamente um terço dos pacientes com SS têm envolvimento extraglandular sistêmico (VIVINO FB, et al., 2019).

Apesar do exposto acima, observa-se uma certa escassez de estudos relatando a frequência, a gravidade ou a morbidade das complicações oculares achadas em pacientes com SS primária, sendo necessário um maior conhecimento sobre a associação entre as mesmas e a SS, dado que algumas delas têm potencial de determinar sérias repercussões clínicas com grande perda da qualidade de vida do paciente (MATHEWS PM, et al., 2015; AKPEK EK, et al., 2015). Tal conhecimento e melhor entendimento é essencial também para o diagnóstico precoce e tratamento efetivo, evitando a ocorrência de sequelas irreversíveis no processo.

Com base no exposto acima, este trabalho tem como objetivo conhecer as taxas de associação entre Síndrome de Sjogren e suas complicações oculares, analisando segundo o protocolo STROBE.

\section{MÉTODOS}

O estudo caracterizou-se por ser uma pesquisa transversal analítica, realizada a partir de junho de 2019 até julho de 2020, que analisou estudos publicados com aprovação prévia do comitê de ética e disponíveis no PubMed, motor de busca de livre acesso à base de dados Medical Literature Analysis and Retrievel System Online (MEDLINE), que associem a SS a complicações oftalmológicas e que, por esta razão, não precisou de nova submissão ao comitê, uma vez que nenhum indivíduo sofreu qualquer tipo de nova intervenção.

Foi adotado um mecanismo de busca sensível para realizar a pesquisa, combinando termos oficiais e sinônimos desta temática. As buscas foram realizadas por meio dos seguintes descritores: "Sjogren's Syndrome/complications" [Mesh]; (cross sectional study); (observational study); (cohort study); (case control), combinados e filtrados para estudos em humanos. Os estudos foram selecionados para compor a amostra a partir da leitura dos títulos e resumos, de forma independente. Foram incluídos estudos observacionais, em qualquer idioma, submetidos desde a criação da base de dados até julho de 2020, assim como foram excluídos os estudos cujo acessos foram impossíveis aos pesquisadores por questão financeira ou logística.

Os artigos selecionados passaram por uma avaliação criteriosa e de qualidade, de acordo com os critérios da iniciativa STROBE, que constitui um conjunto de recomendações para melhorar a qualidade e aumentar a relevância dos relatórios de estudos observacionais (VANDENBROUCKE JP, et al., 2014).

Por abranger diversos desenhos: coorte, caso-controle e estudos transversais observacionais, o STROBE se caracteriza por ser a ferramenta mais amplamente adotada por autores e editores para avaliar a qualidade dos trabalhos científicos. É composta por um checklist com 22 itens, desenvolvidos para recomendar tópicos essenciais em cada seção (título, resumo, introdução, metodologia, resultados, discussão e financiamento) de um estudo com os desenhos abrangidos pela ferramenta. Tal instrumento objetiva contribuir para uma redação mais criteriosa desses estudos, tornando mais fácil a seleção daqueles que vão pautar a tomada de decisão na prática dos profissionais de saúde (PACHECO RL, et al., 2017). 
Desta forma, foram avaliados 6 tópicos referentes aos capítulos dos projetos (título/resumo, introdução, método, resultados, discussão, outras informações), totalizando 23 subtópicos (Tabela 1), que, após a leitura de cada estudo foram, classificados em: 1) Adequado - se preencheu todos, senão a maioria, dos prérequisitos da iniciativa STROBE para aquele capítulo; 2) Inadequado - se não preencheu os pré-requisitos estabelecidos pela iniciativa STROBE para aquele capítulo; 3) Insuficiente - se o artigo não apresentou informações suficientes para análise adequada daquele capítulo, de acordo com a iniciativa STROBE. Os dados colhidos em cada artigo foram tabulados e analisados em planilhas digitais usando o software Microsoft Excel 2016.

\section{RESULTADOS E DISCUSSÃO}

Do total de 723 artigos analisados, 713 não estavam de acordo com os critérios de inclusão e exclusão propostos. Dos 10 artigos concordantes, 3 ainda estão sob análise pois não se encontravam disponíveis na íntegra, exigindo requerimento perante os autores dos mesmos. Portanto 7 artigos foram usados pelo presente estudo (Figura 1).

Figura 1 - Fluxograma de triagem, inclusão, exclusão e seleção de estudos.

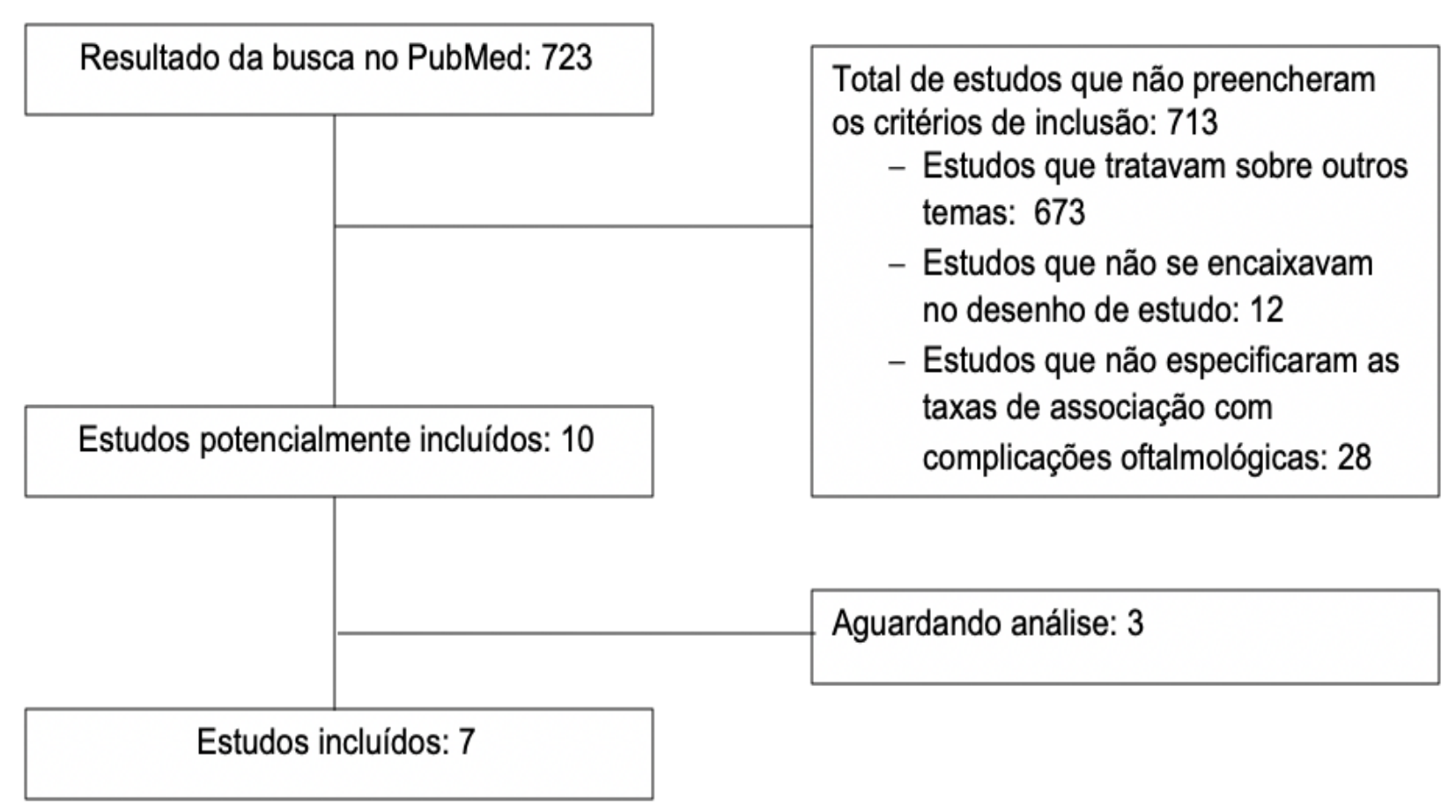

Fonte: Silveira RM, et al., 2020.

Os estudos foram desenvolvidos nos Estados Unidos da América (EUA), Canadá, Noruega e China, tendo como principal público da amostragem pacientes com síndrome de Sjogren primária (QIAO L, et al., 2015; AKPEK EK, et al., 2015; MATHEWS PM, et al., 2015; ACS M, et al., 2018; AQRAWI LA, et al., 2018; CAFFERY B, et al., 2018; LI Z, et al., 2018).

Nesse contexto, a secura ocular foi o sintoma do olho seco com maior associação com síndrome de Sjogren (98.4\%), seguido de irritação e ardor ocular, fotofobia e ceratoconjuntivite. Outras complicações oftalmológicas mais raramente associadas foram uveítes, inflamação orbital, esclerite, vasculite retiniana e neuromielite óptica (Quadro 1). 
Quadro 1- Caracterização dos artigos analisados.

\begin{tabular}{|c|c|c|c|c|c|}
\hline Autor & País & Amostra & População-alvo & Complicações Oftalmológicas & Associação \\
\hline Acs M, et al. (2018) & $\begin{array}{c}\text { Canadá / } \\
\text { EUA }\end{array}$ & 123 pacientes & Geral & $\begin{array}{l}\text { - Olho seco } \\
\text { - Secura ocular } \\
\text { - Irritação ocular } \\
\text { - } \text { Ardor ocular } \\
\text { - } \text { Fotofobia } \\
\text { - } \text { Problemas visuais } \\
\end{array}$ & $\begin{array}{l}-\quad 98.4 \% \\
-\quad 51.2 \% \\
-\quad 44.7 \% \\
-\quad 45.5 \% \\
-\quad 59.3 \% \\
\end{array}$ \\
\hline Aqrawi LA, et al. (2018) & Noruega & 59 pacientes & Síndrome Sjogren Primária & - Olho seco & - $81,1 \%$ \\
\hline Caffery B, et al. (2018) & $\begin{array}{c}\text { Canadá / } \\
\text { EUA }\end{array}$ & 123 pacientes & Geral $\geq 18$ anos & $\begin{array}{l}\text { - } \text { Olho seco } \\
\text { - Secura ocular } \\
\text { - } \text { Irritação ocular } \\
\text { - } \text { Ardor ocular } \\
\text { - } \text { Fotofobia } \\
\text { - } \text { Problemas visuais } \\
\text { - } \text { Ceratoconjuntivite }\end{array}$ & $\begin{array}{l}-\quad 89.4 \% \\
-\quad 42.0 \% \\
-\quad 31.0 \% \\
-\quad 23.0 \% \\
-\quad 30.0 \% \\
-\quad 6.0 \% \\
\end{array}$ \\
\hline Li Z, et al. (2018) & China & 224 pacientes & Síndrome Sjogren Primária & - Olho seco & - $62,5 \%$ \\
\hline Akpek EK, et al. (2015) & EUA & 163 pacientes & Síndrome Sjogren Primária & $\begin{array}{l}\text { - Lesões na córnea } \\
\text { - Lesões na conjuntiva } \\
\text { - Uveítes } \\
\text { - Inflamação orbital } \\
\text { - Esclerite } \\
\text { - Neuromielite óptica } \\
\text { - Vasculite retiniana } \\
\end{array}$ & $\begin{array}{ll} & 11.0 \% \\
& 15,9 \% \\
\text { - } & 3.0 \% \\
\text { - } & 1.8 \% \\
\text { - } & 1.8 \% \\
\text { - } & 1.8 \% \\
\text { - } & 0.6 \% \\
\end{array}$ \\
\hline Mathews PM, et al. (2015) & EUA & 163 pacientes & Síndrome Sjogren Primária & $\begin{array}{l}\text { - Lesões na córnea } \\
\text { - Lesões na conjuntiva } \\
\text { - Uveítes } \\
\text { - Inflamação orbital } \\
\text { - Esclerite } \\
\text { - Neuromielite óptica } \\
\text { - Vasculite retiniana }\end{array}$ & $\begin{array}{ll}-11.0 \% \\
\text { - } 15,9 \% \\
\text { - } 3.0 \% \\
\text { - } 1.8 \% \\
\text { - } 1.8 \% \\
\text { - } 1.8 \% \\
\text { - } & 0.6 \% \\
\end{array}$ \\
\hline Qiao L, et al. (2015) & China & 616 pacientes & Síndrome Sjogren Primária & $\begin{array}{l}\text { - Neuromielite Óptica } \\
\text { - } \text { Perda visual } \\
\text { - Defeito de campo visual } \\
\text { - } \quad \text { Diplopia }\end{array}$ & $\begin{array}{ll}- & 6,9 \% \\
- & 67,4 \% \\
- & 30,2 \% \\
- & 7.0 \%\end{array}$ \\
\hline
\end{tabular}

Fonte: Silveira RM, et al., 2020 
Quanto a avaliação dos artigos segundo os critérios estabelecidos pelo STROBE, observou-se que todos os tópicos se enquadraram majoritariamente na categoria "Adequado"; destes "Introdução", "Título e Resumo" e "Outras informações" demonstraram melhor desempenho, o primeiro com um percentual de $100 \%$ e os dois últimos com 91,67\%. Em relação a inadequação, o tópico "Resultados" apresentou maior percentual (17,14\%), assim como na classificação "Insuficiente" (14,29\%), juntamente com "Título e Resumo", "Discussão" e "Outras informações" (Tabela 1).

Tabela 1 - Classificação dos artigos segundo os 6 tópicos categorizados pela iniciativa STROBE.

\begin{tabular}{ccccccc}
\hline $\begin{array}{c}\text { Classificação/ } \\
\text { Tópico } \\
\text { STROBE }\end{array}$ & $\begin{array}{c}\text { Título e } \\
\text { Resumo } \\
(\mathbf{1})\end{array}$ & $\begin{array}{c}\text { Introdução } \\
\mathbf{( 2 - 3 )}\end{array}$ & $\begin{array}{c}\text { Método } \\
\mathbf{4}-\mathbf{1 2}\end{array}$ & $\begin{array}{c}\text { Resultados } \\
\mathbf{1 3 - 1 7}\end{array}$ & $\begin{array}{c}\text { Discussão } \\
\mathbf{1 8 - 2 1}\end{array}$ & $\begin{array}{c}\text { Outras } \\
\text { Informações } \\
\mathbf{2 2}\end{array}$ \\
\hline Adequado & 12 & 14 & 48 & 24 & 21 & 6 \\
& $(85,71 \%)$ & $(100,00 \%)$ & $(76,19 \%)$ & $(68,57 \%)$ & $(75,00 \%)$ & $(85,71 \%)$ \\
Inadequado & 0 & 0 & 8 & 6 & 3 & 0 \\
& $(0,00 \%)$ & $(0,00 \%)$ & $(12,70 \%)$ & $(17,14 \%)$ & $(10,71 \%)$ & $(00,00 \%)$ \\
Insuficiente & 2 & 0 & 7 & 5 & 4 & 1 \\
& $(14,29 \%)$ & $(0,00 \%)$ & $(11,11 \%)$ & $(14,29 \%)$ & $(14,29 \%)$ & $(14,29 \%)$ \\
\hline \multirow{2}{*}{ TOTAL } & 14 & 14 & 63 & 35 & 28 & 7 \\
& $(100,00 \%)$ & $(100,00 \%)$ & $(100,00 \%)$ & $(100,00 \%)$ & $(100,00 \%)$ & $(100,00 \%)$ \\
\hline
\end{tabular}

Fonte: Silveira RM, et al., 2020

A análise dos subtópicos revelou que "Resumo informativo e equilibrado", "Contexto/ Justificativa (introdução)", "Objetivos (Introdução)", "Participantes (Método)", "Desfecho (Resultados)" e "Resultados principais (Discussão)" foram adequados na totalidade dos artigos avaliados. Os subtópicos "Desenho de estudo (Método)", "Tamanho do estudo (Método)", "Participantes (resultados)" e "Dados descritivos (Resultados)" evidenciaram maior inadequação, observada em 2 artigos avaliados. Já o subtópico "Resultados principais (resultados)" foi o que apresentou maior resultado na classificação "Insuficiente" (Tabela 2).

Tabela 2 - Classificação dos artigos quanto aos 23 subtópicos da iniciativa STROBE.

\begin{tabular}{lccc}
\hline Subtópicos STROBE/Classificação (T=6 artigos) & Adequado & Inadequado & Insuficiente \\
\hline 1a - Desenho do estudo no título e/ou resumo & $5(71,43 \%)$ & $0(0,00 \%)$ & $2(28,57 \%)$ \\
1 b - Resumo informativo e equilibrado & $7(100,00 \%)$ & $0(0,00 \%)$ & $0(0,00 \%)$ \\
2 - Contexto/ Justificativa (Introdução) & $7(100,00 \%)$ & $0(0,00 \%)$ & $0(0,00 \%)$ \\
3 - Objetivos (Introdução) & $7(100,00 \%)$ & $0(0,00 \%)$ & $0(0,00 \%)$ \\
4 - Desenho do estudo (Método) & $5(71,43 \%)$ & $2(28,57 \%)$ & $0(0,00 \%)$ \\
5 - Contexto (Método) & $6(85,71 \%)$ & $1(14,29 \%)$ & $0(0,00 \%)$ \\
6 - Participantes (Método) & $7(100,00 \%)$ & $0(0,00 \%)$ & $0(0,00 \%)$ \\
7 - Variáveis (Método) & $6(85,71 \%)$ & $1(14,29 \%)$ & $0(0,00 \%)$ \\
8 - Fontes de dados/mensuração (Método) & $6(85,71 \%)$ & $0(0,00 \%)$ & $1(14,29 \%)$ \\
9 - Viés (Método) & $2(28,57 \%)$ & $1(14,29 \%)$ & $4(57,14 \%)$ \\
10 - Tamanho do estudo (Método) & $5(71,43 \%)$ & $2(28,57 \%)$ & $0(0,00 \%)$ \\
11 - Variáveis quantitativas (Método) & $5(71,42 \%)$ & $1(14,29 \%)$ & $1(14,29 \%)$ \\
12 - Métodos estatísticos (Método) & $6(85,71 \%)$ & $0(0,00 \%)$ & $1(14,29 \%)$ \\
13 - Participantes (Resultados) & $5(71,43 \%)$ & $2(28,57 \%)$ & $0(0,00 \%)$ \\
14 - Dados descritivos (Resultados) & $5(71,43 \%)$ & $2(28,57 \%)$ & $0(0,00 \%)$ \\
15 - Desfecho (Resultados) & $7(100,00 \%)$ & $0(0,00 \%)$ & $0(0,00 \%)$ \\
16 - Resultados principais (Resultados) & $1(14,29 \%)$ & $1(14,29 \%)$ & $5(71,42 \%)$ \\
17 - Outras análises (Resultados) & $6(85,71 \%)$ & $1(14,29 \%)$ & $0(0,00 \%)$ \\
18 - Resultados principais (Discussão) & $7(100,00 \%)$ & $0(0,00 \%)$ & $0(0,00 \%)$ \\
19 - Limitações (Discussão) & $5(71,42 \%)$ & $1(14,29 \%)$ & $1(14,29 \%)$ \\
20 - Interpretação (Discussão) & $6(85,71 \%)$ & $1(14,29 \%)$ & $0(0,00 \%)$ \\
21 - Generalização (Discussão) & $3(42,86 \%)$ & $1(14,28 \%)$ & $3(42,86 \%)$ \\
22 - Financiamento (Outras informações) & $6(85,71 \%)$ & $0(0,00 \%)$ & $1(14,29 \%)$ \\
\hline TOTAL & $\mathbf{1 1 1}(80,43 \%)$ & $\mathbf{9 ( 6 , 5 2 \% )}$ & $\mathbf{1 8 ( 1 3 , 0 4 \% )}$ \\
\hline
\end{tabular}

Fonte: Silveira RM, et al., 2020. 
Até onde se tem ciência, o presente trabalho constitui-se como o primeiro mapeamento realizado analisando os estudos que associam Síndrome de Sjogren e suas complicações oftalmológicas. Este mapeamento proporciona um olhar panorâmico a respeito dos distúrbios abordados e das pesquisas realizadas sobre 0 assunto, além de permitir uma melhor categorização qualitativa destas com uma abordagem segundo a iniciativa STROBE.

De acordo com o objetivo primário do presente trabalho, a análise dos artigos publicados na base de dados PubMed que fizessem associação entre SS e suas complicações oculares, foi constatado que os artigos selecionados se propuseram a estudar diferentes complicações do quadro ocular da SS e apresentaram algumas discordâncias em seus achados.

As associações entre síndrome de Sjogren e suas complicações oftalmológicas, encontradas nos trabalhos analisados, são destacadas na Quadro 1. Tais associações mostram-se heterogêneas, sendo perceptível uma grande ocorrência de olho seco com 98,4 e 89,4 \% de taxas de associação nos artigos de Acs M, et al. (2018) e Caffery B, et al. (2018), ao mesmo tempo que se observou taxas de associação menores, a exemplo de $81,1 \%$ e 62,5\% (AQRAWI LA, et al., 2018; LI Z, et al., 2018).

Isso pode ser explicado pelos diferentes tamanhos amostrais dos estudos (59 e 224 pacientes) e diferentes perfis das populações entre os três países em que estes ocorreram, sendo a ocorrência de Síndrome de Sjögren mais comum em mulheres caucasianas (LI Z, et al., 2018).

Outro fator importante a ser levado em conta é a inexistência de um único teste definitivo para a determinação de olho seco. Com a existência de variados testes, é fundamental a associação entre dados da história clínica, sintomas e achados do exame físico para melhor caracterização da síndrome, o que desfavorece uma padronização no diagnóstico da doença do olho seco (BERNARDI FR, et al., 2015).

Nesse sentido, foi observado uma prevalência de 5-50\% quando se falava em "sintomas do olho seco" e uma prevalência maior que $75 \%$ em algumas populações quando se falava de "sinais de olho seco" (STAPLETON F, et al., 2017). Tais resultados, também heterogêneos, se encontram em consonância com os encontrados pela presente pesquisa.

Ainda na Quadro 1, é possível perceber, nos estudos de Akpek EK, et al. (2015) e Mathews PM, et al. (2015), a ocorrência de graves complicações com potencial de grande comprometimento visual e impacto na qualidade de vida, a exemplo de uveíte $(3,0 \%)$, de esclerite $(1,8 \%)$, de neuromielite ótica $(1,8 \%)$ e de vasculite retiniana $(0.6 \%)$. Nesse sentido, o estudo de Qiao $\mathrm{L}$, et al mostra que $6,9 \%$ de uma população de 616 pacientes evoluíram com neuromielite ótica, e, dentro destes, 67,4\% evoluíram com perda total da visão.

Apesar de serem taxas de associação relativamente pequenas, é necessário entender que elas são diretamente proporcionais ao tempo de evolução do quadro clínico da doença (BECKMAN K, et al., 2016). Tendo em vista isso, observa-se a importância do diagnóstico da Síndrome de Sjogren em seus momentos iniciais, a fim de evitar uma evolução grave e irreversível do quadro clínico.

É importante ressaltar também que os estudos de Acs M, et al. (2018); Caffery B, et al. (2018) e os de Akpek EK, et al. (2015) e Mathews PM, et al. (2015), foram feitos com base na mesma amostra populacional de pacientes. Isso pode explicar a falta de outras discordâncias e de maior heterogeneidade entre os dados obtidos. Além disso, não há grande discrepância entre o tamanho das populações estudadas, sendo a menor de 59 e a maior de 616 .

Tem-se também que o perfil de pacientes estudados foi similar entre os artigos em aspectos epidemiológicos importantes, comumente englobando, em sua maioria, mulheres na faixa etária da meia idade. Tais fatos podem ser caracterizados como um possível viés de seleção, não apresentando a real associação geral entre a SS e suas complicações oftalmológicas.

Outro possível viés para o presente estudo reside no fato de que os pacientes estudados nos artigos analisados pertenciam, em sua totalidade, a centros de atenção terciária. Como centros terciários são responsáveis pelo acompanhamento e tratamento da enfermidade específica, é possível que haja uma maior 
associação às complicações avaliadas se comparado a estudos populacionais com comunidades extensas, que não necessariamente tenham história de acompanhamento em tais centros, podendo mostrar taxas de associação menores.

Por fim, a falta de qualidade metodológica avaliada de acordo com os critérios da iniciativa STROBE foi um fator muito percebido pelos pesquisadores durante a realização do presente trabalho. Devido a isso, muitos artigos não puderam ser analisados ou completamente compreendidos, tendo as taxas de associação omitidas ou colocadas de maneira incorreta e/ou equivocada, impossibilitando a devida comparação e possibilitando, por vezes, a super/subestimação de alguns resultados.

\section{STROBE}

Os tópicos da iniciativa STROBE que se mostraram mais adequados na maioria dos estudos analisados foram "Título/Resumo", "Introdução" e "Outras informações". Tal fato pode ser atribuído à simplicidade exigida para a redação dos mesmos, sendo tópicos que não requerem um grande conhecimento técnico e um amplo domínio teórico da metodologia do estudo. Além disso, os dois primeiros possuíam mais de um subtópico, dos quais bastava preencher o exigido em um deles para considerá-lo como adequado.

Os tópicos "Metodologia" e "Resultados" foram os que figuraram com maior inadequação na avaliação, o que retrata a dificuldade que a maioria dos autores têm em fundamentar suas pesquisas nestes que são pontos fundamentais para o bom entendimento por parte do leitor e para a relevância do estudo perante a comunidade científica. A metodologia de um trabalho, quando mal explicada ou executada, coloca em xeque a real validade e aplicabilidade do estudo feito. Os resultados mal elaborados prejudicam o entendimento dos achados da pesquisa, impossibilitando a mesma de ser utilizada como referência para outros trabalhos, a exemplo deste.

Nesse contexto, ainda na "Metodologia", o "Viés" foi o subtópico que mais se mostrou insuficiente ao ser avaliado, demonstrando a dificuldade existente em conseguir se determinar com precisão os fatores que podem ser caracterizados como tais. Estudos com viés produzem resultados que diferem sistematicamente da verdade. É importante que o leitor saiba quais medidas foram tomadas durante a realização de um estudo para reduzir o potencial e o risco da ocorrência de viés.

Quanto aos resultados o subtópico avaliado mais negativamente foi o de "resultados principais". Os autores devem explicar todos os fatores de confusão em potencial que foram considerados e os critérios para excluir ou incluir variáveis em modelos estatísticos. As decisões sobre exclusão ou inclusão de variáveis devem ser orientadas pelo conhecimento, ou suposições explícitas, a fim de validar os resultados do trabalho e facilitar a compreensão do leitor (VANDENBROUCKE JP, et al., 2014).

Em relação ao tópico "Discussão", o subtópico que se mostrou mais inadequado durante a avaliação foi "Generalização", que se caracteriza por ser a discussão da validade externa dos resultados. Constatou-se que em metade dos trabalhos não houve esta abordagem, interferindo diretamente na interpretação dos dados pelo leitor, que pode erroneamente generalizar um resultado específico e adquirir um entendimento errado acerca do assunto estudado.

"Outras informações", que teve como subtópico "Financiamento", se mostrou adequado na grande maioria dos projetos. Isto mostra que os autores deram importância para os aspectos financeiros da realização de seus estudos, transparecendo ao leitor como foi o custeio de determinada pesquisa, mesmo quando este foi feito com recurso próprio dos autores, facilitando possíveis reproduções futuras e testando a sua viabilidade financeira. Além disso, informa qualquer potencial fonte de viés do trabalho ligado ao custeio financeiro deste.

Este trabalho apresentou como limitação o fato de realizar a busca em apenas uma base de dados (PubMed), restringindo o rol de estudos analisados a uma parcela da totalidade de artigos produzidos nesse tema. Além de abranger largamente a associação entre Síndrome Sjogren e complicações oftalmológicas, tendo como resultado pesquisas que não necessariamente buscavam a mesma linha de raciocínio, impossibilitando, portanto, a comparação direta entre as associações. 


\section{CONSIDERAÇÕES FINAIS}

A associação entre síndrome de Sjogren e suas complicações oculares mostrou-se legítima dada as diversas estimativas de efeitos encontradas nos trabalhos, nas suas devidas proporções com amostras, no geral significantes, distribuídas em várias regiões do mundo. Esta também se mostrou heterogênea: há uma maior associação a quadros oftalmológicos iniciais, como a doença do olho seco; e menores taxas de associação a complicações mais graves como uveíte, esclerite, neuromielite ótica e vasculite retiniana, todas relacionadas de forma diretamente proporcional ao tempo de doença. A partir desta associação, ressalta-se a importância do diagnóstico da síndrome de Sjogren no início do quadro clínico, a fim de evitar o agravamento de suas manifestações e a ocorrência de complicações que podem trazer consequências irreversíveis para o paciente. Ademais, concluiu-se, a partir da análise dos artigos estudados, seguindo o protocolo STROBE, que muitos autores ainda não possuem pleno conhecimento da descrição metodológica, da análise dos resultados e da exposição correta da validade de generalização do trabalho, acarretando ao leitor o risco de uma leitura e de uma interpretação incorreta do trabalho estudado. Dessa forma são necessários mais estudos sobre o tema, sendo realizados de maneira padronizada entre as diferentes amostras, a fim de que possa ser feita uma comparação mais fidedigna entre estas, além de uma maior difusão de conhecimentos a respeito da correta elaboração de artigos científicos.

\section{REFERÊNCIAS}

1. ACS M, et al. Customary practices in the monitoring of dry eye disease in Sjogren's syndrome. Journal of optometry, 2018; 11(4): 232-241.

2. AKPEK EK, et al. Ocular and systemic morbidity in a longitudinal cohort of Sjögren's syndrome. Ophthalmology, 2015; 122(1): 56-61.

3. AKPEK EK, et al. Ocular and systemic morbidity in a longitudinal cohort of Sjögren's Syndrome. Ophthalmology, 2015; 122(1): 56-61.

4. AKPEK EK, et al. Sjögren's Syndrome: More Than Just Dry Eye. Cornea, 2019, v. 38(5): 658.

5. ALMEIDA JÚNIOR ADD. Modulação autonômica cardíaca em mulheres na pós-menopausa com síndrome do olho seco Dissertação (Mestrado em Ciências). Universidade de São Paulo, São Paulo, 2019; 95p.

6. AQRAWI LA, et al. Severity of clinical dry eye manifestations influences protein expression in tear fluid of patients with primary Sjögren's syndrome. PloS one, 2018; 13(10): e0205762.

7. BECKMAN K, et al. Making the diagnosis of Sjögren's syndrome in patients with dry eye. Clinical ophthalmology (Auckland, NZ), 2016; 10: 43.

8. BERNARDI FR, et al. Olho seco: diagnóstico e manejo / Dry eye: diagnosis and management. Acta méd. (Porto alegre), 2015; 36 (7).

9. BETIM FCM, et al. Da Descoberta às Perpectivas da Síndrome de Sjögren: uma Revisão de Literatura. Revista Eletrônica Biociências, Biotecnologia e Saúde. 2016; 5(14): 20-31.

10. CAFFERY B, et al. Sjogren's syndrome in optometric practices in North America. Contact Lens and Anterior Eye, 2018; 41(6): 518-526.

11. CENDÓN CHAPELA S. Síndrome de Sjögren Primário: implicações na cavidade oral. (Dissertação) Tese de Doutorado. Universidade Fernando Pessoa, Porto, 2016; 83p.

12. LI Z, et al. Prevalence, severity, and predictors of dry eye and dry mouth in Chinese patients with primary Sjögren syndrome. Clinical Rheumatology, 2018; 37(11): 2971-2979.

13. MALLMANN CS. Avaliação estereológica da glândula salivar menor em pacientes com suspeita de Síndrome de Sjögren. Dissertação (Mestrado em Imunologia Básica e Aplicada) - Universidade Federal do Amazonas, Manaus, 2015; 85p.

14. MATHEWS PM, et al. Ocular complications of primary Sjögren syndrome in men. American journal of ophthalmology, $2015 ; 160(3): 447-452$.

15. MAVRAGANI CP, et al. O tratamento da Síndrome de Sjögren. Portal do Envelhec, 2019; 1-13.

16. MILANI LMJ, et al. Análise morfológica e bioquímica das alterações vasculares na Síndrome de Sjögren. Brazilian Oral Research. 2017; 31-524.

17. PACHECO RL, et al. Guidelines para publicação de estudos científicos. Parte 2: Como publicar estudos observacionais (coorte, caso-controle e transversal). Diagn. tratamento, 2017; 22(3): 121-126.

18. QIAO L, et al. The clinical characteristics of primary Sjogren's syndrome with neuromyelitis optica spectrum disorder in China: a STROBE-compliant article. Medicine, 2015; 94(28): 1145.

19. RIBEIRO MVMR, et al. Eficácia do uso de colírios sem preservativos comparados com colírios com preservativos no tratamento do olho seco: uma revisão sistemática. Arquivos Brasileiros de Oftalmologia, 2019; 82(5): 436-445.

20. STAPLETON F, et al. TFOS DEWS II Epidemiology Report. The ocular surface, 2017; 15(3): 334-365.

21. VALIM V, et al. Recomendações para o tratamento da síndrome de Sjögren. Rev. Bras. Reumatol. $2015 ; 55(5): 46$.

22. VANDENBROUCKE JP, et al. Strengthening the Reporting of Observational Studies in Epidemiology (STROBE): explanation and elaboration. International journal of surgery, 2014; 12(12): 1500-1524.

23. VIVINO FB, et al. Sjogren's syndrome: An update on disease pathogenesis, clinical manifestations and treatment. Clinical immunology, 2019; 203: 81. 